\title{
Deep Astrometric Standards
}

\section{Platais ${ }^{1}$, A. L. Fey ${ }^{2}$, S. Frey ${ }^{3}$, S. G. Djorgovski ${ }^{4}$, C. Ducourant ${ }^{5}$, Ž. Ivezić ${ }^{6}$, A. Rest ${ }^{7}$, C. Veillet $^{8}$, R. F. G. Wyse ${ }^{1}$ and N. Zacharias ${ }^{2}$}

${ }^{1}$ Johns Hopkins University, Department of Physics \& Astronomy, Baltimore, MD 21218, USA email: imants, wyse@pha.jhu.edu

${ }^{2}$ US Naval Observatory, Washington DC, USA

${ }^{3}$ FÖMI Satellite Geodetic Observatory, Hungary; ${ }^{4}$ Caltech, Pasadena, CA 91125, USA

${ }^{5}$ Observatoire de Bordeaux, Floirac, France

${ }^{6}$ University of Washington, Seattle, WA 98155, USA

${ }^{7}$ Harvard University, Cambridge, MA 02138, USA; ${ }^{8}$ CFHT, Kamuela, HI 96743, USA

\begin{abstract}
The advent of next-generation telescopes with very wide fields-of-view creates a need for deep and precise reference frames for astrometric calibrations. The Deep Astrometric Standards (DAS) program is designed to establish such a frame, by providing absolute astrometry at the 5-10 mas level in four $10 \mathrm{deg}^{2}$ Galactic fields, to a depth of $V=25$. The source of our basic reference frame is the UCAC2 catalog, significantly improved by additional observations, and new VLBI positions of radio-loud and optically visible QSOs. We describe all the major steps in the construction of the DAS fields and provide the current status of this project.
\end{abstract}

Keywords. astrometry, catalogs

\section{Introduction}

When astronomers refer to the term "astrometric standard", there could be differences in the perception of what this entails. For a quick astrometric calibration of images (i.e., deriving celestial coordinates in the system of ICRS), there are a few all-sky optical catalogs readily available. For many applications in astronomy these are sufficient, except for astrometry itself. Why? Because none of these catalogs can match the accuracy of formal image centering, now reaching 5-10 mas with the best ground-based imaging telescopes, and around 1 mas with the Hubble Space Telescope. This argument gains another dimension when confronted with large aperture telescopes and huge CCD mosaic cameras, such as the planned LSST facility (Tyson 2002). For these new facilities, some of the key science requirements aimed at unlocking the secrets of dark matter and dark energy mandate that this accuracy of image centering is not lost in manipulation of images, such as co-adding images obtained with significant dithers. From the standpoint of astrometry, there are two main issues with modern imaging facilities: 1) the fragmented detector plane (a mosaic of solid-state devices) and 2) the considerable geometric distortions in the focal plane. The combination of these two issues requires a very complex plate model involving hundreds of unknowns. Such a complex model is not readily described by ad hoc solutions, particularly at high Galactic latitudes where few reference stars are available. To address these issues, we proposed in 2006 to set up Deep Astrometric Standards (DAS; Platais et al. 2006) which should allow us a significant simplification of the plate model, reducing the number of unknowns from hundreds potentially down to only three basic constants - zeropoint, scale (stretch), and rotation. This enables robust and reliable 
equatorial solutions anywhere on the sky. In this contribution we report on the status of this project.

\section{Properties of the DAS fields}

Considering the specifications of Pan-STARRS (Kaiser 2004) and LSST facilities and their operational needs, four DAS fields have been established (Platais et al. 2006). Each DAS field covers a $\sim 10 \mathrm{deg}^{2}$ semi-circular area with a projected depth down to $V=25$.

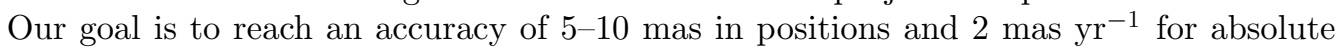
proper motions. At optical wavelengths, such high positional accuracies over a relatively large area at $V>12$ are yet to be achieved. Our strategy in reaching of this goal is to tie the optical positions directly into the International Celestial Reference Frame (ICRF) by densifying locally the ICRF itself. This provides an exceptional opportunity to ensure that metrics of a reference frame defined by the DAS is invariant to one part in $\sim 100,000$ over one degree of arc.

The specified depth of DAS fields requires at least 3-4m class telescopes equipped with a CCD mosaic imager, now a commonly available detector for astrometry. Platais et al. (2002) have demonstrated that the National Optical Astronomy Observatory's (NOAO) CCD mosaic imagers are suitable for astrometry (despite some initial scepticism in the community), being stable at the 10 mas level over a single lunation period. This level of stability is one of the key factors to make the DAS project feasible. Another critical component in the realization of DAS is access to a good reference frame. Currently the best relatively dense optical reference frame is the UCAC2 (Zacharias et al. 2004) but even this should be locally improved by acquiring new observations with automated meridian circles and other means. One of the biggest concerns is that the reference frame represented by the UCAC2 could be locally biased in some way that would then propagate into the DAS positions. New ICRF sources in the DAS fields should play a key role in identifying, quantifying and eliminating this kind of problem.
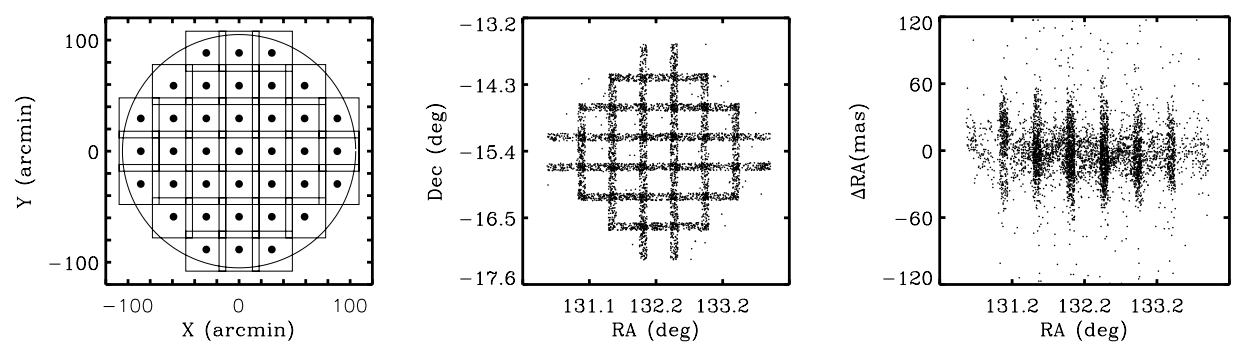

Figure 1. Left panel: Pointing layout of a DAS field with the NOAO $4 \mathrm{~m}$ telescopes and their CCD mosaic imagers. In total, 37 partially overlapping (overlap $\Delta=6^{\prime}$ ) pointings are necessary to fill in a 3.5-diameter field; Middle panel: Spatial distribution of stars in the frame overlap area (a few points elsewhere are artifacts); Right panel: Coordinate differences of the overlap region stars in the Hydra field from one complete pass (37 pointings) in a recent run. The rms of the scatter is about 25 mas, still dominated by residual systematic errors that have yet to be accounted for.

\section{Optical imaging}

The locations of the DAS fields require observations from both hemispheres. The first DAS field in Hydra was observed in February 2006 with the Cerro Tololo Inter-American 
Observatory's (CTIO) Blanco $4 \mathrm{~m}$ telescope $\left(\mathrm{FOV}=36^{\prime} \times 36^{\prime}\right)$. In the same year another DAS field in Sagittarius was completed at CTIO. As indicated in Fig. 1, a total of 37 partially overlapping pointings are necessary to fill a DAS field. The wide DAS magnitude range of $10<V<25$ means that a set of $10 \mathrm{~s}, 100 \mathrm{~s}$, and $600 \mathrm{~s}$ exposures are required at each pointing in the Sloan $g$ and $i$ filters. In total, the average number of CCD frames per field is about 350. In a similar manner we have completed the northern DAS field in Ophiuchus, using the Kitt Peak Mayall 4m telescope. The remaining field in Gemini-Orion-Taurus is scheduled for observations in early 2008 with the $3.6 \mathrm{~m}$ CanadaFrance-Hawaii Telescope (CFHT). We have obtained the pixel positions for objects in all Hya and Sgr mosaic frames. We use the software developed for NOAO surveys such as MSCPIPE and PHOTPIPE; these include standard manipulations for CCD data and DoPHOT object detection, photometry, and astrometry (Schechter et al. 1993) - all streamlined and with minimal supervision. Preliminary results are shown in Fig. 1.

\section{Local improvements to the UCAC}

Our plan is to use a dense optical reference frame - the UCAC - to calibrate our CCD mosaic frames and refine the constants of the geometric distortions across the field. This catalog is known to have small systematic errors, on the order of 5-10 mas, at the epoch of the CCD observations around yr 2000. More important, however, are the proper motions needed to extrapolate to current epochs. As indicated by Zacharias et al. (2004), there are problems with the positions from photographic plates, which are used to derive proper motions at $V>12$. To avoid such complications, we are obtaining new epoch observations with a monolithic 111M pixel CCD camera attached to the USNO Twin Astrograph's "red lens" tube. In parallel, we have already observed two DAS fields with the Bordeaux automatic CCD meridian circle, in a time delay integration mode (e.g., Rapaport et al. 2001). Except for the final coordinate zeropoint, such observations are independent of the reference catalog and should help to minimize local systematics in UCAC. The coordinate zeropoint will be set by the optical counterparts of new ICRF sources, which will also provide the means to check the system of equatorial coordinates.

\section{Ancillary observations}

Conventional reductions of observations at two or more epochs produce relative proper motions. To make them absolute, the positions should be linked to an extragalactic reference frame represented by QSOs and/or galaxies with well-defined cores. Unfortunately, background QSOs are rare even with wide fields-of-view. We have initiated a new search for bright QSOs in the DAS fields, using the Palomar-Quest 161M-pixel camera, operated in a drift scan mode, through SDSS griz filters (see http://palquest.org). In color-color diagrams the QSO candidates show up as outliers from the stellar sequence, although this domain is also occupied by hot white dwarfs and some other Galactic objects with peculiar spectral energy distributions. Spectroscopic confirmation is therefore required to establish a list of bona fide QSOs and for this we will utilize the Palomar 200 inch telescope, with the Double Spectrograph.

The DAS fields, with one exception, lack previous observations in the SDSS griz filters. Secondary standards of calibrated griz photometry are now being set up in the DAS fields using the $0.5 \mathrm{~m}$ Photometric Telescope at Apache Point Observatory, New Mexico, and the US Naval Observatory's $1 \mathrm{~m}$ telescope at the Flagstaff station. These standards will be used to calibrate the DAS instrumental $g, i$ magnitudes. 


\section{Local densification of the ICRF}

One of the most innovative aspects of DAS is its direct link to the ICRF. This requires considerable effort to find optically visible compact radio sources and to measure their positions using the technique of VLBI phase referencing. As a first step, in each DAS field we select $\sim 25$ unresolved strong sources $\left(S_{20 \mathrm{~cm}}>50 \mathrm{mJy}\right)$ from the 20-cm NRAO VLA Sky Survey (NVSS; Condon et al. 1998). Each source is then observed with the Very Large Array (VLA) at 3.5 and $6 \mathrm{~cm}$, to measure its radio spectrum and verify that it is indeed compact. Extragalactic radio sources show a flat-spectrum core, with extended emission in the form of steep-spectrum jet components. Typically about $50 \%$ of our initial sample survives after the VLA observations. Secondly, the surviving compact sources are observed with a VLBI facility, such as the European VLBI Network (EVN). Our first phase-referencing EVN observations at $6 \mathrm{~cm}$ were conducted in November 2006. In the Gemini-Orion-Taurus field they produced seven bona fide detections with peak brightness ranging from $\sim 5 \mathrm{mJy}$ to $46 \mathrm{mJy}$ per beam (Frey et al. 2007). The few existing CFHT deep frames indicate that four EVN detections have a reasonably bright optical counterpart but for two other EVN detections any optical counterpart is fainter than the limiting magnitude. We plan to extend the search for more sources in the GOT field at lower flux densities $\left(50>S_{20 \mathrm{~cm}}>20 \mathrm{mJy}\right)$ since the south-east portion of this field so far has produced no VLBI detections.

\section{Project status}

With lots of enthusiasm but with essentially zero dedicated funding we were able to obtain the first epoch optical imaging in all four DAS fields and test all vital steps of the DAS concept. The VLA observations of candidate compact sources are completed in three DAS fields. One lesson we have learned from the VLA and VLBI observations is that the fainter radiosources $\left(S_{20 \mathrm{~cm}}>20 \mathrm{mJy}\right)$ should also be considered, as indicated by successful VLBI detections of additional sources from the GB6 catalog (Gregory et al. 1996). The first DAS catalog is expected to appear in the second part of 2008.

\section{Acknowledgements}

IP gratefully acknowledges support from the US National Science Foundation through grant AST 04-06689 to Johns Hopkins University. SF acknowledges support from the Hungarian Scientific Research Fund (OTKA T046097). We thank Allyn Smith and Douglas Tucker for obtaining initial data for the secondary SDSS standard in a DAS field.

\section{References}

Condon, J. J., Cotton, W. D., Greissen, E. W., et al. 1998, AJ, 115, 1693

Frey, S., Platais, I., \& Fey, A. L. 2007, in Proc. 18th European VLBI for Geodesy and Astrometry Working Meeting, eds. J. Boehm, A. Pany, H. Schuh, p. 111

Gregory, P. C., Scott, W. K., Douglas, K., \& Condon, J. J. 1996, ApJS, 103, 427

Kaiser, N. 2004, Proc. SPIE, 5489, 11

Platais, I., Wyse, R. F. G., \& N. Zacharias 2003, PASP, 118, 107

Platais, I., Kozhurina-Platais, V., Girard, et al. 2002, AJ, 124, 601

Rapaport, M., Le Campion, J.-F., Soubiran, C., et al. 2001, A\&GA, 376, 325

Schechter, P., Mateo, M., \& Saha, A. 1993, PASP, 105, 1342

Tyson, J. A. 2002, Proc. SPIE, 4836, 10

Zacharias, N., Urban, S. E., Zacharias, M. I., Wycoff, G. L., Hall, D. M., Monet, D. G., \& Rafferty, T. J. 2004, AJ, 127, 3043 